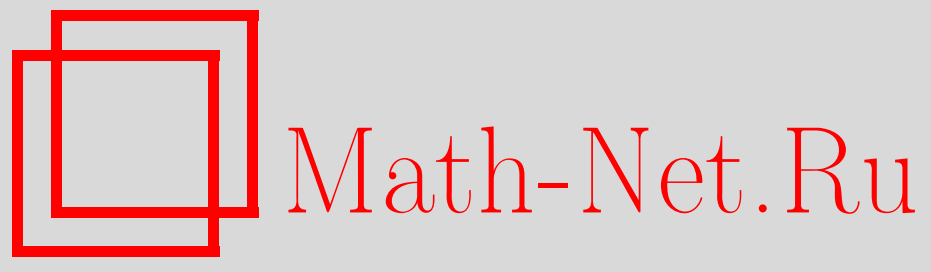

В. В. Пржиялковский, Инварианты Громова-Виттена трехмерных многообразий Фано рода 6 и рода $8, M a$ тем. сб., 2007, том 198, номер 3, 145-158

DOI: https://doi.org/10.4213/sm3773

Использование Общероссийского математического портала Math-Net.Ru подразумевает, что вы прочитали и согласны с пользовательским соглашением http://www.mathnet.ru/rus/agreement

Параметры загрузки:

IP : 44.207 .124 .84

26 апреля 2023 г., 18:16:22

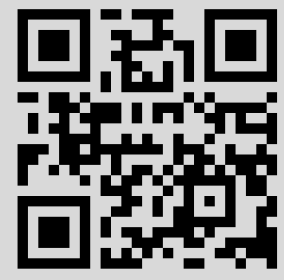




\section{В. В. Пржиялковский}

\section{Инварианты Громова-Виттена трехмерных многообразий Фано рода 6 и рода 8}

Работа посвящена доказательству для многообразий $V_{10}$ и $V_{14}$ гипотезы Голышева о модулярности уравнений $D 3$ для гладких трехмерных многообразий Фано с группой Пикара $\mathbb{Z}$. А именно, найдены считающие матрицы примарных двухточечных инвариантов многообразий $V_{10}$ и $V_{14}$ с помощью метода нахождения инвариантов Громова-Виттена полных пересечений в многообразиях, для которых эти инварианты (частично) известны.

Библиография: 33 названия.

Для того чтобы найти двухточечные инварианты Громова-Виттена многообразий $V_{10}$ и $V_{14}$, в работе использован следующий метод. Согласно Ш. Мукаи эти многообразия являются полными пересечениями в грассманианах (теорема 3.1.1). Найдем производящий ряд их одноточечных инвариантов с потомками (так называемый $I$-ряд). Воспользуемся квантовой теоремой Лефшеца (см. следствие 4.2.2), чтобы найти $I$-ряд для $V_{10}$ и $V_{14}$. После этого по аксиоме дивизора и топологической рекурсии найдем полиномиальные выражения для коэффициентов $I$-ряда через двухточечные примарные инварианты (предложение 6.2.2). Наконец, вычислим значения двухточечных инвариантов, обратив эти выражения.

В $\S 1$ даются определения инвариантов Громова-Виттена и пространств модулей кривых и отображений рода 0 с отмеченными точками. В $\S 2$ формулируется гипотеза Голышева. Третий параграф посвящен $I$-рядам для грассманианов. Четвертый параграф содержит квантовую теорему Лефшеца. В пятом содержатся необходимые соотношения между одно- и многоточечными инвариантами. И наконец, в шестом доказывается основная теорема этой работы (теорема 6.1.1), в которой находятся считающие матрицы многообразий $V_{10}$ и $V_{14}$.

Библиография. Инварианты Громова-Виттена были введены для подсчета чисел кривых различного рода, лежащих на многообразиях и пересекающих данные гомологические классы. Однако потом это понятие было расширено. Аксиоматическое определение примарных инвариантов было дано в [1]. В дальнейшем они были построены и обобщены в [2] и [3].

Сведение инвариантов Громова-Виттена гиперповерхности к инвариантам объемлющего многообразия (зеркальная формула), основной из известных на сегодня методов вычисления квантовых когомологий, принадлежит, по-видимому, А. Гивенталю (см., к примеру, [4]). В этой работе она используется в форме, полученной А. Гатманном (см. [5]). Гипотеза Хори-Вафа (т.е. I-ряд для грассманианов) была доказана А. Бертрамом, И. Циоканн-Фонтанином и

Работа выполнена при поддержке Российского фонда фундаментальных исследований (гранты №№ 04-01-00613, 05-01-00353) и Программы поддержки ведущих научных школ РФ (грант № НШ-489.2003.1) 
Б. Кимом (см. [6]). А. Бертрамом и Х. Клеем, а также Я.-П. Ли и Р. Пандхарипанде были получены соотношения, позволяющие сводить многоточечные инварианты к одноточечным (см. [7] и [8]).

Трехмерные многообразия Фано родов 6 и 8 изучались в работах [9]-[19]. Инварианты Громова-Виттена для таких многообразий, соответствующие прямым, были найдены в [19], см. также [15]; число коник на многообразии рода 6, проходящих через точку, было найдено в [16].

Инварианты Громова-Виттена трехмерных многообразий Фано начали изучаться в [20]. Квантовые когомологии некоторых многообразий были найдены в [21] (некоторые раздутия проективного пространства и квадрики), [22], [23] (торические многообразия), [24] (расслоения над $\mathbb{P}^{n}$ ) и [25] (некоторые полные пересечения).

Гипотеза модулярности трехмерных гладких многообразий Фано с группой Пикара $\mathbb{Z}$ была сформулирована В. Голышевым в [26].

Соглашения и обозначения. Основным полем считается поле комплексных чисел $\mathbb{C}$. Под грассманианом $G(r, n)$ будет подразумеваться многообразие $r$-мерных линейных подпространств в $n$-мерном линейном пространстве. Под инвариантами Громова-Виттена подразумеваются инварианты рода 0, т.е. соответствующие рациональным кривым. Алгебру когомологий $H^{*}(X, \mathbb{Q})$ многообразия $X$ будем обозначать через $H^{*}(X)$. Двойственный по Пуанкаре класс к классу $\gamma \in H^{*}(X)$ на многообразии $X$ будем обозначать через $\gamma^{\vee}$. Подалгебру алгебраических циклов в $H^{*}(X)$ будем обозначать через $H_{\mathrm{alg}}^{*}(X)$. Подмножество классов эффективных кривых в $H_{2}(X, \mathbb{Z})$ будем обозначать через $H_{2}^{+}(X)$.

\section{§ 1. Основные определения}

Рассмотрим гладкое проективное многообразие $X$ с группой Пикара $\mathbb{Z}$ такое, что $-K_{X}$ численно эффективен.

Мы ограничимся только теми определениями и аксиомами, которые будут использоваться в дальнейшем.

\section{1. Пространства модулей.}

ОПРЕДЕЛЕНИЕ 1.1.1. Родом кривой $C$ называется число $h^{1}\left(\mathscr{O}_{C}\right)$.

Легко видеть, что связная кривая имеет род 0 тогда и только тогда, когда она является деревом рациональных кривых.

ОПРЕДЕЛЕНИЕ 1.1.2. Связная кривая $C$ с $n \geqslant 0$ отмеченными точками $p_{1}, \ldots, p_{n} \in C$ называется предстабильной, если она имеет особенности не хуже обыкновенных двойных точек, а $p_{1}, \ldots, p_{n}$ - различные гладкие точки (см. [27; гл. III, п. 2.1]). Отображение $f: C \rightarrow X$ связной кривой рода 0 с $n$ отмеченными точками называется стабильным, если $C$ - предстабильная кривая, а на каждой ее стягиваемой компоненте лежат как минимум три отмеченные или особые точки ([27; гл. V, п. 1.3.2]).

Другими словами, стабильное отображение связной кривой - это отображение, имеющее лишь конечное число инфинитезимальных автоморфизмов. 
ОПРЕДЕЛЕНИЕ 1.1.3. Семейством стабилъных отображений (над схемой $S$ ) кривых рода 0 с $n$ отмеченными точками называется набор

$$
\left(\pi: \mathscr{C} \rightarrow S, p_{1}, \ldots, p_{n}, f: \mathscr{C} \rightarrow X\right),
$$

где $\pi$ - плоское проективное отображение с $n$ сечениями $p_{1}, \ldots, p_{n}$, геометрические слои $\left(\mathscr{C}_{s}, p_{1}(s), \ldots, p_{n}(s)\right)$ которого являются предстабильными кривыми рода 0 с $n$ отмеченными точками такими, что ограничение $\left.f\right|_{\mathscr{C}_{s}}$ на каждый из этих слоев является стабильным отображением.

Два семейства над $S$

$$
\left(\pi: \mathscr{C} \rightarrow S, p_{1}, \ldots, p_{n}, f\right), \quad\left(\pi^{\prime}: \mathscr{C}^{\prime} \rightarrow S, p_{1}^{\prime}, \ldots, p_{n}^{\prime}, f^{\prime}\right)
$$

называются изоморфными, если существует изоморфизм $\tau: \mathscr{C} \rightarrow \mathscr{C}^{\prime}$ такой, что $\pi=\pi^{\prime} \circ \tau, p_{i}^{\prime}=\tau \circ p_{i}, \quad f=f^{\prime} \circ \tau$.

Пусть $\beta \in H_{2}^{+}(X)$. Рассмотрим следующий (контравариантный) функтор $\overline{\mathscr{M}}_{n}(X, \beta)$ из категории (комплексных алгебраических) схем в категорию множеств. Пусть $\overline{\mathscr{M}}_{n}(X, \beta)(S)$ - это множество классов изоморфизмов семейств стабильных отображений кривых рода 0 с $n$ отмеченными точками $(\pi: \mathscr{C} \rightarrow S$, $\left.p_{1}, \ldots, p_{n}, f\right)$ таких, что $f_{*}\left(\left[\mathscr{C}_{s}\right]\right)=\beta$, где $\left[C_{s}\right]-$ фундаментальный класс $C_{s}$.

ОПРЕДЕЛЕНИЕ 1.1.4. Пространством модулей отображений в $X$ кривых рода 0 класса $\beta \in H_{2}^{+}(X)$ с $n$ отмеченными точками $\bar{M}_{n}(X, \beta)$ называется стек Делиня-Мамфорда (см. [27; гл. V, п. 5.5]), являющийся грубым пространством модулей, представляющим $\overline{\mathscr{M}}_{n}(X, \beta)$.

$\bar{M}_{n}(X, \beta)$ - компактный гладкий стек, локально являющийся фактором неособого многообразия по конечной группе, так что на нем существует теория пересечений (см. [28]). В общем случае $\bar{M}_{n}(X, \beta)$ имеет “неправильную" размерность, так что его принято снабжать виртуальным фундаментальным классом $\left[\bar{M}_{n}(X, \beta)\right]^{\mathrm{virt}}$ виртуальной размерности

$$
\operatorname{vdim} \bar{M}_{n}(X, \beta)=\operatorname{dim} X-\operatorname{deg}_{K_{X}} \beta+n-3 .
$$

Пусть $X$ - выпуклое многообразие (т.е. для любого отображения $\mu: \mathbb{P}^{1} \rightarrow X$ выполнено $\left.H^{1}\left(\mathbb{P}^{1}, \mu^{*}(T X)\right)=0\right)$. Тогда $\bar{M}_{n}(X, \beta)$ - проективное нормальное многообразие чистой размерности $\operatorname{dim} X-\operatorname{deg}_{K_{X}} \beta+n-3$, так что виртуальный фундаментальный класс совпадает с обычным. Подробнее о его конструкции можно прочесть в [27; гл. VI, п. 1.1].

\section{2. Инварианты Громова-Виттена. Рассмотрим отображения}

$$
\mathrm{ev}_{i}: \bar{M}_{n}(X, \beta) \rightarrow X, \quad \mathrm{ev}_{i}\left(C ; p_{1}, \ldots, p_{n}, f\right)=f\left(p_{i}\right) .
$$

Пусть $\pi_{n+1}: \bar{M}_{n+1}(X, \beta) \rightarrow \bar{M}_{n}(X, \beta)-$ морбизм, забывающий точку $p_{n+1}$ (и стягивающий компоненты, которые становятся нестабильными при забывании $\left.p_{n+1}\right)$, а $\sigma_{i}: \bar{M}_{n}(X, \beta) \rightarrow \bar{M}_{n+1}(X, \beta)$ - сечение, соответствующее отмеченной точке $p_{i}$. Образом кривой $\left(C ; p_{1}, \ldots, p_{n}, f\right)$ при отображении $\sigma_{i}$ будет кривая $\left(C^{\prime} ; p_{1}, \ldots, p_{n+1}, f^{\prime}\right)$, где $C^{\prime}=C \cup C_{0}, C_{0} \simeq \mathbb{P}^{1}, C_{0}$ и $C$ пересекаются в (не отмеченной на $C^{\prime}$ ) точке $p_{i}$, а точки $p_{n+1}$ и $p_{i}$ лежат на $C_{0}$. При этом $f^{\prime}$ стягивает $C_{0}$ в точку, а $\left.f^{\prime}\right|_{C}=f$ (см. рис. 1$)$.

Пусть $L_{i}=\sigma_{i}^{*} \omega_{\pi_{n+1}}$, где $\omega_{\pi_{n+1}}$ - относительный дуализирующий пучок $\pi_{n+1}$. Слоем $L_{i}$ над точкой $\left(C ; p_{1}, \ldots, p_{n}, f\right)$ является $T_{p_{i}}^{*} C$. Пусть кокасательный линейный класс равен $\psi_{i}=c_{1}\left(L_{i}\right) \in H^{2}\left(\bar{M}_{n}(X, \beta)\right)$. 


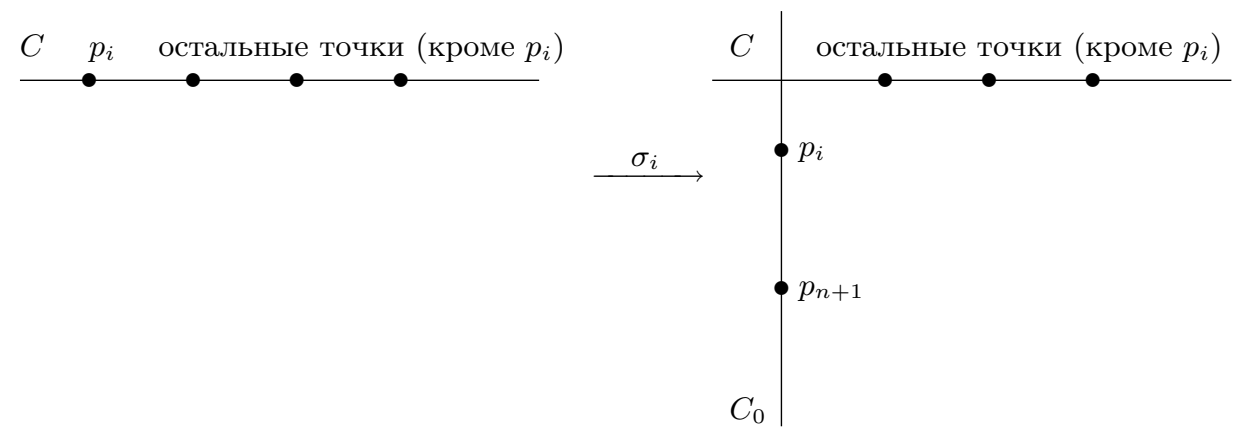

Рис. 1

ОПРЕДЕЛЕНИЕ 1.2 .1 [27; гл. VI, п. 2.1]. Пусть $\gamma_{1}, \ldots, \gamma_{n} \in H^{*}(X), d_{1}, \ldots, d_{n}$ - целые неотрицательные числа. Инвариантом Громова-Виттена (коррелятором) с потомками называется число

$$
\left\langle\tau_{d_{1}} \gamma_{1}, \ldots, \tau_{d_{n}} \gamma_{n}\right\rangle_{\beta}=\mathrm{ev}_{1}^{*} \gamma_{1} \cdot \psi_{1}^{d_{1}} \ldots \mathrm{ev}_{n}^{*} \gamma_{n} \cdot \psi_{n}^{d_{n}} \cdot\left[\bar{M}_{n}(X, \beta)\right]^{\mathrm{virt}},
$$

если $\sum \operatorname{codim} \gamma_{i}+\sum d_{i}=\operatorname{vdim} \bar{M}_{n}(X, \beta)$, и 0 в противном случае. Число $\sum d_{i}$ называется степенъю инварианта относительно потомков. Инварианты степени 0 называются примарными (и символы $\tau_{0}$ опускаются).

При таком определении аксиомы инвариантов Громова-Виттена становятся их свойствами. Приведем некоторые из них.

- Аксиома дивизора [27; гл. VI, п. 5.4]. Пусть $\gamma_{0} \in H^{2}(X)$. Тогда

$$
\begin{aligned}
& \left\langle\gamma_{0}, \tau_{d_{1}} \gamma_{1}, \ldots, \tau_{d_{n}} \gamma_{n}\right\rangle_{\beta}=\left(\gamma_{0} \cdot \beta\right)\left\langle\tau_{d_{1}} \gamma_{1}, \ldots, \tau_{d_{n}} \gamma_{n}\right\rangle_{\beta} \\
& +\sum_{k, d_{k} \geqslant 1}\left\langle\tau_{d_{1}} \gamma_{1}, \ldots, \tau_{d_{k}-1}\left(\gamma_{0} \cdot \gamma_{k}\right), \ldots, \tau_{d_{n}} \gamma_{n}\right\rangle_{\beta}
\end{aligned}
$$

за исключением случая $\beta=0$ и $n=2$. В этом случае

$$
\left\langle\gamma_{0}, \gamma_{1}, \gamma_{2}\right\rangle_{0}=\gamma_{0} \cdot \gamma_{1} \cdot \gamma_{2} \cdot[X]
$$

- Аксиома фундаментального класса [27; гл. VI, п. 5.1]. Пусть 1 - фундаментальный класс $X$. Тогда

$$
\left\langle\mathbf{1}, \tau_{d_{1}} \gamma_{1}, \ldots, \tau_{d_{n}} \gamma_{n}\right\rangle_{\beta}=\sum_{k, d_{k} \geqslant 1}\left\langle\tau_{d_{1}} \gamma_{1}, \ldots, \tau_{d_{k}-1} \gamma_{k}, \ldots, \tau_{d_{n}} \gamma_{n}\right\rangle_{\beta}
$$

за исключением случая $\beta=0$ и $n=2$. В этом случае

$$
\left\langle\mathbf{1}, \gamma_{1}, \gamma_{2}\right\rangle_{0}=\gamma_{1} \cdot \gamma_{2} \cdot[X]
$$

ЗАмечАниЕ 1.2.2. Инварианты Громова-Виттена будут рассматриваться в случае ранга группы Пикара равного 1. В этом случае класс кривой $\beta \in H_{2}^{+}(X)$ характеризуется своей степенью $d$ (относительно положительной образующей группы Пикара), поэтому вместо $\langle\ldots\rangle_{\beta}$ мы часто будем писать $\langle\ldots\rangle_{d}$. Для случая большего ранга необходимо использовать мультистепень. 
1.3. $I$-ряды [5]. Пусть $R \subset H^{*}(X)$ - самодвойственная по Пуанкаре алгебра (т.е. для любого класса $\gamma \in R$ имеем $\gamma^{\vee} \in R$ ). Пусть $1=\gamma_{0}, \ldots, \gamma_{N}-$ базис в $R$. Пусть $\beta \in H_{2}^{+}(X)$ - эффективная кривая степени $d$ (относительно положительной образующей группы Пикара). Положим

$$
I_{d, R}^{X}=I_{\beta, R}^{X}=\sum_{i, j}\left\langle\tau_{i} \gamma_{j}\right\rangle_{\beta} \gamma_{j}^{\vee}
$$

ОПРЕДЕЛЕНИЕ 1.3.1 [5]. I-ряд $I_{R}^{X}$ задается следующей формулой:

$$
I_{R}^{X}=\sum_{d \geqslant 0} I_{d, R}^{X} \cdot q^{d} .
$$

Для $R=H^{*}(X) I$-ряд $I_{R}^{X}$ мы будем обозначать просто через $I^{X}$; для $R=$ $H_{\text {alg }}^{*}(X)$ - через $I_{\text {alg. }}^{X}$. Если $Y \subset X-$ полное пересечение, а $\pi: H^{*}(X) \rightarrow H^{*}(Y)-$ естественный гомоморфизм ограничения, то для $R=\pi\left(H^{*}(X)\right)$ ряд $I_{R}^{X}$ будем обозначать через $I_{\text {rest }}^{X}$.

ОПРЕДЕЛЕНИЕ 1.3.2. Пусть $I \in H^{*}(X) \otimes \mathbb{C}[[q]]$. Положим

$$
I=\sum_{0 \leqslant i \leqslant N} \gamma_{i} \otimes I^{(i)}(q) \text {. }
$$

Член $I^{(0)}$ мы будем обозначать через $I_{H^{0}}$.

\section{§ 2. Считающие матрицы трехмерных многообразий Фано}

\section{1. Считающие матрицы.}

ОПРЕДЕЛЕНИЕ 2.1.1 [26]. Пусть $X$ - гладкое трехмерное многообразие Фано с одномерной группой Пикара, $K=-K_{X}$. Считающей матрищей называется матрица $A \in \operatorname{Mat}(4 \times 4)$ вида

$$
\left[\begin{array}{cccc}
a_{00} & a_{01} & a_{02} & a_{03} \\
1 & a_{11} & a_{12} & a_{13} \\
0 & 1 & a_{22} & a_{23} \\
0 & 0 & 1 & a_{33}
\end{array}\right]
$$

нумерация столбцов и строк которой начинается с нуля, а элементы задаются следующим образом:

$$
a_{i j}=\frac{\left\langle K^{3-i}, K^{j}, K\right\rangle_{j-i+1}}{\operatorname{deg} X}=\frac{j-i+1}{\operatorname{deg} X} \cdot\left\langle K^{3-i}, K^{j}\right\rangle_{j-i+1}
$$

(степень антиканоническая).

Легко видеть, что матрица $A$ симметрична относительно побочной диагонали: $a_{i j}=a_{3-j, 3-i}$. По определению $a_{i j}=0$ при $j-i+1<0$. Если $j-i+1=0$, то $a_{i j}=1$, так как $a_{i j}$ в этом случае - просто число точек пересечения $K^{3-i}, K^{j}$ и $K$, которое, очевидно, равно $\operatorname{deg} X ; a_{00}=a_{33}=0$. Остальные коэффициенты $a_{i j}$ - это "ожидаемые" числа рациональных кривых степени $j-i+1$, пересекающих $K^{3-i}$ и $K^{j}$, умноженные на $(j-i+1) / \operatorname{deg} X$, за одним исключением. А именно, по аксиоме дивизора

$$
a_{01}=2 \cdot(2 \cdot \operatorname{ind}(X) \cdot[\text { число коник, проходящих через общую точку] }) \text {. }
$$


2.2. Гипотеза Голышева. Вместо одной матрицы нам будет удобней рассматривать семейство матриц $A^{\lambda}=A+\lambda E$, где $E$ - единичная матрица. Таким образом, элемент семейства $A^{\lambda}$ определяется заданием шести параметров пяти различных $\mathrm{GW}$-инвариантов $a_{i j}$ и $\lambda$.

Рассмотрим одномерный тор $\mathbb{G}_{m}=\operatorname{Spec} \mathbb{C}\left[t, t^{-1}\right]$ и дифференциальный оператор $D=t \frac{\partial}{\partial t}$ на нем. Построим семейство матриц $M^{\lambda}$ с элементами $m_{k l}^{\lambda}$, равными

$$
m_{k l}^{\lambda}= \begin{cases}0, & \text { если } k>l+1, \\ 1, & \text { если } k=l+1, \\ a_{k l}^{\lambda} \cdot(D t)^{l-k+1}, & \text { если } k<l+1 .\end{cases}
$$

Теперь рассмотрим семейство дифференциальных операторов

$$
\widetilde{L}^{\lambda}=\operatorname{det}_{\text {right }}\left(D E-M^{\lambda}\right)
$$

где det $_{\text {right }}$ означает “правый определитель", т.е. определитель, полученный путем разложения по правому столбиу. Все миноры также разлагаются по правому столбцу. Разделив $\widetilde{L}^{\lambda}$ слева на $D$, получим семейство операторов $L^{\lambda}$, т.е. $\widetilde{L}^{\lambda}=D L^{\lambda}$.

ОПРЕДЕЛЕНИЕ 2.2.1 [26; п. 1.8]. Уравнение из семейства $L^{\lambda}[\Phi(t)]=0$ называется считающим уравнением D3.

ГиПотеЗА 2.2.2 (В. Голышев [26]). Для трехмерного гладкого многообразия Фано с группой Пикара $\mathbb{Z}$ решение считающего уравнения D3 модулярно. Конкретнее, пусть $X$ - такое многообразие, $i_{X}-$ его индекс, а $N=$ $\operatorname{deg} X /\left(2 i_{X}^{2}\right)$. Тогда в семействе считающих уравнений для $X$ есть такое уравнение $L^{\lambda X}[\Phi(t)]=0$, решением которого является ряд Эйзенштейна веса 2 на $X_{0}(N)$.

Основываясь на этой гипотезе, В. Голышев в [26] составил список матриц, гипотетически совпадающих со считающими матрицами трехмерных многообразий Фано. Для проверки гипотезы необходимо найти все такие матрицы.

\section{§ 3. I-ряды и грассманианы}

\section{1. Теорема Мукаи.}

Теорема 3.1 .1 [18]. Гладкое трехмерное многообразие Фано $V_{10}$ рода 6 ( и антиканонической степени 10) является сечением грассманиана $G(2,5)$ линейным пространством коразмерности 2 и квадрикой в плюккеровом вложении.

Гладкое трехмерное многообразие Фано $V_{14}$ рода 8 (и антиканонической степени 14) является сечением грассманиана $G(2,6)$ линейным пространством коразмерности 5 в плюккеровом вложении.

Эта теорема является частным случаем общей теоремы Мукаи, описывающей гладкие трехмерные многообразия Фано с группой Пикара $\mathbb{Z}$ в терминах сечений пучков на грассманианах. 


\subsection{I-ряды для грассманианов.}

Tеорема 3.2.1 (гипотеза Хори-Вафа [29; Appendix A], доказательство см. в [6]). Пусть $x_{1}, \ldots, x_{r}-$ корни Чюеня расслоения $S^{*}$ на $G=G(r, n)$, двойственного $к$ тавтологическому подрасслоению, где $r>1$. Тогда

$$
I^{G}=\sum_{d \geqslant 0}(-1)^{(r-1) d} \sum_{d_{1}+\cdots+d_{r}=d} \frac{\prod_{1 \leqslant i<j \leqslant r}\left(x_{i}+d_{i}-x_{j}-d_{j}\right)}{\prod_{1 \leqslant i<j \leqslant r}\left(x_{i}-x_{j}\right) \prod_{i=1}^{r} \prod_{l=1}^{d_{i}}\left(x_{i}+l\right)^{n}} q^{d} .
$$

В случае $r=1$ (т.е. для проективного пространства)

$$
I^{\mathbb{P}^{n-1}}=\sum_{d \geqslant 0} \prod_{i=1}^{d} \frac{q^{d}}{(H+i)^{n}}
$$

где $H$ - класс, двойственный гиперплоскому сечению.

СледСтвиЕ 3.2.2 [6; предложение 3.5]. Свободный член ряда $I^{G}$ для $G=$ $G(2, n)$ равен

$$
\sum_{d \geqslant 0} \frac{q^{d}}{(d !)^{n}} \frac{(-1)^{d}}{2} \sum_{m=0}^{d}\left(\begin{array}{c}
d \\
m
\end{array}\right)^{n}(n(d-2 m)(\gamma(m)-\gamma(d-m))+2),
$$

əдe

$$
\gamma(m)=\sum_{j=1}^{m} \frac{1}{j}, \quad \gamma(0)=0
$$

\section{§ 4. Квантовая теорема Лефшеца}

Многообразие $X$, как и раньше, предполагается гладким проективным с группой Пикара $\mathbb{Z}$.

\subsection{I-ряд гиперповерхности.}

ЛЕмма 4.1.1 [30; лемма 5.5], [8; доказательство леммы 1]. Пусть $Y \subset X-$ полное пересечение, $\varphi: H^{*}(X) \rightarrow H^{*}(Y)$ - гомоморфизм ограничения. Пусть

$$
\widetilde{\gamma}_{1} \in \varphi\left(H^{*}(X)\right)^{\perp}, \quad \gamma_{2}, \ldots, \gamma_{l} \in \varphi\left(H^{*}(X)\right) .
$$

Тогда для любого $\beta \in \varphi\left(H_{2}^{+}(X)\right) \subset H_{2}^{+}(Y)$ инвариант Громова-Виттена на $Y$ вида

$$
\left\langle\tau_{d_{1}} \widetilde{\gamma}_{1}, \tau_{d_{2}} \gamma_{2}, \ldots, \tau_{d_{l}} \gamma_{l}\right\rangle_{\beta}
$$

равен нулю.

Таким образом, по аксиоме дивизора (1) и лемме 4.1.1 $I^{Y}=I_{\text {rest }}^{Y}$.

ЗАмЕчАНИЕ 4.1.2. В отличие от одноточечных инвариантов двухточечные инварианты, соответствующие классам в $\varphi\left(H^{*}(X)\right)^{\perp}$, могут быть ненулевыми (см. [25; предложение 1]). 


\section{2. Зеркальная формула.}

Теорема 4.2.1 (зеркальная формула [5; следствие 1.13]). Рассмотрим гиперповерхность $Y \subset X$ такую, что $-K_{Y} \geqslant 0$. Тогда существуют рядь $R(q) \in H^{*}(X)[[q]]$ u $S(q) \in H^{*}(X)[[q]]$ maкuе, что

$$
\sum_{\beta} \prod_{i=0}^{Y \cdot \beta}(Y+i) \cdot I_{\beta}^{X} \cdot q^{Y \cdot \beta}=R(q) \cdot \sum_{\beta} I_{\beta}^{Y} \cdot \widetilde{q}^{Y \cdot \beta},
$$

где $\widetilde{q}=q \cdot e^{S(q)}$.

А. Гатманн в [5; определение 1.11 и лемма 1.12] дал описание этих рядов.

В случае многообразий Фано зеркальная формула упрощается.

СледСтвиЕ 4.2.2. Пусть

$$
I=\operatorname{ind} Y \in \mathbb{N}
$$

- индекс многообразия Фано $Y$. Тогда если $Y$ - полное пересечение гиперповерхностей $Y_{1}, \ldots, Y_{k}$ степеней $d_{1}, \ldots, d_{k}$ в $X$, mо

$$
\sum_{\beta} \prod_{j=1}^{k} \prod_{i=0}^{Y_{j} \cdot \beta}\left(Y_{j}+i\right) \cdot I_{\beta}^{X} \cdot q^{Y \cdot \beta}=e^{\alpha_{Y} \cdot q^{\operatorname{deg} Y}} \cdot \sum_{\beta} I_{\beta}^{Y} \cdot q^{Y \cdot \beta}
$$

где $\alpha_{Y}=\prod d_{i} ! \cdot I_{1, H^{0}}^{X}$, если $I=1, u \alpha_{Y}=0$, если $I \geqslant 2$.

ДокАЗАТЕЛЬСтво. Из вида рядов $R(q)$ и $S(q)$ в случае многообразий Фано следует, что $R(q)=e^{\alpha_{Y} \cdot q^{\operatorname{deg} Y}}$, а $S(q)=0$. Для $I>1$ имеем $R(q)=1$. Чтобы найти $\alpha_{Y}$ для индекса 1 , заметим, что по соображениям размерности $\left\langle H^{\operatorname{dim} Y}\right\rangle_{l}=0$, где $H$ - гиперплоское сечение $X$. Сравнив коэффициенты формулы (3) при $q^{\operatorname{deg} Y}$ и $H^{k}$, получим

$$
\alpha_{Y}=\prod_{i=1}^{k} d_{k} ! \cdot I_{1, H^{0}}^{X},
$$

что и требовалось доказать.

ЗАмЕчАНИЕ 4.2.3. Гипотеза 2.2.2 может быть усилена следующим образом. Положим $\alpha_{Y}=\lambda_{Y}$. Тогда решением уравнения $L^{\lambda_{X}}[\Phi(t)]=0$ является ряд Эйзенштейна веса 2 на $X_{0}(N)$.

\section{§5. Выражение одноточечных инвариантов через примарные двухточечные}

ОПРЕДЕЛЕНИЕ 5.1. Подалгебра $R \in H^{*}(Y)$ называется квантово самодвойственной, если для всех $\gamma \in R, \mu \in R^{\perp}$ и $\beta \in H_{2}^{+}(Y)$ выполнено $\gamma^{\vee} \in R$ и $\langle\gamma, \mu\rangle_{\beta}=0$.

ПреДЛОЖениЕ 5.2. Для каждых $n, I \in \mathbb{N}, k, d \in \mathbb{Z}_{\geqslant 0}$ существует многочлен $f_{k}^{d} \in \mathbb{Q}\left[a_{i, j}\right], 0 \leqslant i, j \leqslant n, j-i+1 \leqslant d$, такой, что верно следующее. Рассмотрим многообразие Фано $Y$ размерности $n$ и индекса I такое, что подалгебра $R \in H^{*}(Y)$, порожденная $-K_{Y}=I H$, квантово самодвойственна. Тогда

$$
\left\langle\tau_{k} H^{d+n-2-k}\right\rangle_{d}=f_{k}^{d}\left(a_{i j}\right),
$$


әде

$$
a_{i, j}=\frac{1}{\operatorname{deg} Y}\left\langle H^{n-i}, H^{\beta}, H\right\rangle_{j-i+1}=\frac{j-i+1}{\operatorname{deg} Y}\left\langle H^{n-i}, H^{j}\right\rangle_{j-i+1} .
$$

ДокАЗАтЕЛьство. Стратегия нахождения $f_{k}^{d}$ такова: по аксиоме дивизора выразим данный одноточечный инвариант через трехточечные с потомками, а потом с помощью топологической рекурсии выразим эти трехточечные инварианты через примарные двухточечные.

Применяя аксиому дивизора для $H$

$$
\left\langle\tau_{k} H^{d+n-2-k}\right\rangle_{d}=\frac{1}{d} \cdot\left(\left\langle H, \tau_{k} H^{d+n-2-k}\right\rangle_{d}-\left\langle\tau_{k-1} H^{d+n-1-k}\right\rangle_{d}\right),
$$

легко получить, что

$$
\left\langle\tau_{k} H^{d+n-2-k}\right\rangle_{d}=\frac{1}{d} \sum_{i=0}^{k} \frac{(-1)^{i}}{d^{i}}\left\langle H, \tau_{k-i} H^{d+i+n-2-k}\right\rangle_{d} .
$$

Далее, по топологической рекурсии (см., например, [31; следствие 1.3])

$$
\begin{aligned}
& \left\langle H^{a}, \tau_{k} H^{d+n-1-a-k}\right\rangle_{d} \\
& =\frac{1}{d}\left(\sum_{d_{1} \leqslant d}\left\langle H^{d_{1}-d+1+a}, \tau_{k-1} H^{d+n-1-a-k}\right\rangle_{d_{1}} \cdot a_{d_{1}-d+a+1, a}\right. \\
& \left.\quad-\left\langle H^{a}, \tau_{k-1} H^{d+n-a-k}\right\rangle_{d}\right) .
\end{aligned}
$$

Эту формулу также легко упростить. Рекурсивно выражая последнее слагаемое в правой части, получаем

$$
\begin{aligned}
& \left\langle H^{a}, \tau_{k} H^{d+n-1-a-k}\right\rangle_{d} \\
& =\sum_{\substack{i=1, \ldots, k \\
d_{1} \leqslant d}} \frac{(-1)^{i+1}}{d^{i}} a_{d_{1}-d+1+a, a}\left\langle H^{d_{1}-d+1+a}, \tau_{k-i} H^{d+n-2-a-k+i}\right\rangle_{d_{1}} \\
& \quad+\frac{(-1)^{k}}{d^{k}}\left\langle H^{a}, H^{d+n-a}\right\rangle_{d} .
\end{aligned}
$$

ЗАмечАниЕ 5.3. Мы предположили, что $\operatorname{Pic} Y=\mathbb{Z}$ и $R$ порождено $H$ для простоты, так как мы будем использовать это в дальнейшем. Аналогичные выражения могут быть найдены так же, как и в доказательстве предложения 5.2, и в общем случае с единственным условием $R \cap H^{2}(Y) \neq \varnothing$.

Пример 5.4. Рассмотрим гладкое многообразие Фано $Y$ индекса 1 и размерности 3 , алгебраические когомологии которого порождены образующей группы Пикара $H$. Тогда

- свободный член $I$-ряда:

1) $\left\langle H^{3}\right\rangle_{2}=\operatorname{deg} Y \cdot \frac{a_{01}}{4}$;

2) $\left\langle\tau H^{3}\right\rangle_{3}=\operatorname{deg} Y \cdot\left(\frac{a_{11} a_{01}}{18}+\frac{a_{02}}{27}\right)$;

3) $\left\langle\tau_{2} H^{3}\right\rangle_{4}=\operatorname{deg} Y \cdot\left(\frac{a_{01}^{2}}{64}+\frac{a_{11}^{2} a_{01}}{96}+\frac{7 a_{11} a_{02}}{576}+\frac{a_{01} a_{12}}{128}+\frac{a_{03}}{256}\right)$; 
- линейный по $H$ член $I$-ряда:

1) $\left\langle H^{2}\right\rangle_{1}=\operatorname{deg} Y \cdot a_{11}$

2) $\left\langle\tau H^{2}\right\rangle_{2}=\operatorname{deg} Y \cdot\left(\frac{a_{11}^{2}}{4}+\frac{a_{12}}{8}-\frac{a_{01}}{4}\right)$;

3) $\left\langle\tau_{2} H^{2}\right\rangle_{3}=\operatorname{deg} Y \cdot\left(\frac{5 a_{11} a_{01}}{108}+\frac{a_{11}^{3}}{18}+\frac{a_{11} a_{12}}{12}-\frac{2 a_{02}}{81}\right)$;

4) $\left\langle\tau_{3} H^{2}\right\rangle_{4}=\operatorname{deg} Y \cdot\left(\frac{13 a_{11}^{2} a_{01}}{576}+\frac{17 a_{11} a_{02}}{1728}-\frac{a_{03}}{256}-\frac{3 a_{01}^{2}}{128}+\frac{a_{11}^{4}}{96}\right.$

$$
\left.+\frac{a_{12}^{2}}{256}+\frac{a_{11}^{2} a_{12}}{32}\right) .
$$

Таким образом,

$$
\begin{aligned}
I^{Y}=1 & +a_{11} q+\left(\frac{a_{01}}{4}+\left(\frac{a_{11}^{2}}{4}+\frac{a_{12}}{8}-\frac{a_{01}}{4}\right) H\right) q^{2}+\left(\left(\frac{a_{11} a_{01}}{18}+\frac{a_{02}}{27}\right)\right. \\
& \left.+\left(\frac{5 a_{11} a_{01}}{108}+\frac{a_{11}^{3}}{18}+\frac{a_{11} a_{12}}{12}-\frac{2 a_{02}}{81}\right) H\right) q^{3} \\
& +\left(\left(\frac{a_{01}^{2}}{64}+\frac{a_{11}^{2} a_{01}}{96}+\frac{7 a_{11} a_{02}}{576}+\frac{a_{01} a_{12}}{128}+\frac{a_{03}}{256}\right)\right. \\
& \left.+\left(\frac{13 a_{11}^{2} a_{01}}{576}+\frac{17 a_{11} a_{02}}{1728}-\frac{a_{03}}{256}-\frac{3 a_{01}^{2}}{128}+\frac{a_{11}^{4}}{96}+\frac{a_{12}^{2}}{256}+\frac{a_{11}^{2} a_{12}}{32}\right) H\right) q^{4} \\
& +\cdots\left(\bmod H^{2}\right) .
\end{aligned}
$$

\section{§6. Основная теорема}

\section{1. Полные пересечения в грассманианах.}

Теорема 6.1.1. Считающие матрицы многообразий $V_{10}$ u $V_{14}$ совпадают с предсказанными в [26] и равны

1) для многообразия $V_{10}$

$$
M\left(V_{10}\right)=\left[\begin{array}{cccc}
0 & 156 & 3600 & 33120 \\
1 & 10 & 380 & 3600 \\
0 & 1 & 10 & 156 \\
0 & 0 & 1 & 0
\end{array}\right]
$$

сдвиг $\alpha_{V_{10}}$ равен 6;

2) для многообразия $V_{14}$

$$
M\left(V_{14}\right)=\left[\begin{array}{cccc}
0 & 64 & 924 & 5936 \\
1 & 5 & 140 & 924 \\
0 & 1 & 5 & 64 \\
0 & 0 & 1 & 0
\end{array}\right]
$$

сдвиг $\alpha_{V_{14}}$ равен 4. 
ДокАЗАТЕЛЬСтво. По теореме 3.1.1 эти многообразия - полные пересечения в $G(2,5)$ (для $\left.V_{10}\right)$ и $G(2,6)$ (для $\left.V_{14}\right)$.

Пусть $H$ - эффективная образующая группы Пикара грассманиана $G=$ $G(r, n)$. Положим $I^{G}=I_{H^{0}}^{G}(q)+I_{H^{1}}^{G}(q) \cdot H\left(\bmod H^{4}(G)\right)$, т.е.

$$
I^{G}=I_{H^{0}}^{G}(q)+I_{H^{1}}^{G}(q) \cdot H+\widetilde{I}
$$

где $\widetilde{I} \in H^{>2}(G)$.

По следствию 3.2 .2

$$
\begin{aligned}
& I_{H^{0}}^{G(2,5)}=1+3 q+\frac{19}{32} q^{2}+\frac{49}{2592} q^{3}+\frac{139}{884736} q^{4}+\cdots \\
& I_{H^{0}}^{G(2,6)}=1+4 q+\frac{3}{4} q^{2}+\frac{95}{5832} q^{3}+\frac{865}{11943936} q^{4}+\cdots
\end{aligned}
$$

Пользуясь теоремой 3.2.1, рассмотрим $I$-ряды грассманианов $G(2,5)$ и $G(2,6)$ как ряды от элементарных симметрических функций $x_{1}+x_{2}$ и $x_{1} x_{2}$ от корней Чженя $x_{1}$ и $x_{2}$ расслоения, двойственного к тавтологическому подрасслоению. Тогда $I_{H^{1}}^{G}$ - это коэффициент при линейной симметрической функции $x_{1}+x_{2}$. Таким образом,

$$
\begin{aligned}
& I_{H^{1}}^{G(2,5)}=10 q+\frac{105}{32} q^{2}+\frac{3115}{23328} q^{3}+\frac{6875}{5308416} q^{4}+\cdots, \\
& I_{H^{1}}^{G(2,6)}=15 q+\frac{609}{128} q^{2}+\frac{6197}{46656} q^{3}+\frac{528737}{764411904} q^{4}+\cdots .
\end{aligned}
$$

По следствию $4.2 .2 \alpha_{V_{10}}=6$ и $\alpha_{V_{14}}=4$. По формуле (3), взятой по модулю $H^{2}$,

$$
\begin{aligned}
I^{V_{10}}=1 & +10 H q+\left(39+\frac{67}{2} H\right) q^{2}+\left(220+\frac{3200}{9} H\right) q^{3} \\
& +\left(\frac{6291}{4}+\frac{89387}{48} H\right) q^{4}+\cdots\left(\bmod H^{2}\right), \\
I^{V_{14}}=1 & +5 H q+\left(16+\frac{31}{4} H\right) q^{2}+\left(2+\frac{1031}{18} H\right) q^{3} \\
& +\left(230+\frac{14863}{96} H\right) q^{4}+\cdots \quad\left(\bmod H^{2}\right) .
\end{aligned}
$$

Легко видеть, что выражения из примера 5.4 позволяют восстановить коэффициенты считающей матрицы многообразия $Y$ из ряда $I^{Y}\left(\bmod H^{2}\right)$.

6.2. Выражение примарных двухточечных инвариантов через одноточечные. Метод нахождения двух- (и более) точечных инвариантов через одноточечные, использованный в теореме 6.1.1, работает и в общем случае. А именно, рассмотрим многообразие $Y$ размерности $n$ и квантово самодвойственную подалгебру $R \subset H^{*}(Y)$ с базисом $\left\{\gamma_{0}, \ldots, \gamma_{N}\right\}$. Пусть $d \in \mathbb{N}$, $\left\langle\tau_{i} \gamma_{j}\right\rangle_{k}=f_{i j}^{k}\left(\left\langle\gamma_{t}, \gamma_{r}\right\rangle_{s}\right)$ (см. замечание 5.3). Пусть известны одноточечные инварианты $Y$ и двухточечные примарные инварианты, соответствующие кривым степени не больше $d$. Тогда одноточечные инварианты, соответствующие кривым степени $d+1$, с помощью функций $f_{i, j}^{d+1}$ линейно выражаются через двухточечные примарные инварианты, соответствующие кривым степени $d+1$. 
Более того, можно выбрать такой набор $\{(i, j)\}$ (где $i \in \mathbb{Z}_{\geqslant 0}, 0 \leqslant j \leqslant N$, $i+j=d+n-1)$, что система линейных уравнений $\left\{f_{i j}^{d+1}\left(\left\langle\gamma_{k}, \gamma_{l}\right\rangle_{d+1}\right)=\left\langle\tau_{i} \gamma_{j}\right\rangle_{d+1}\right\}$ задается невырожденной верхнетреугольной матрицей. Таким образом, индукцией по $d$ можно найти полиномиальные выражения для двухточечных инвариантов через одноточечные. Этот метод обобщает следующую теорему.

ТЕОРема 6.2.1 [7; теорема 5.2]. Пусть $R \subset H^{*}(X)$ - подалгебра, порожденная образующей группь Пикара $X$, a $I^{X}=I_{R}^{X}$. Тогда инварианты ГромоваВиттена вида $\left\langle\tau_{i_{1}} \gamma_{1}, \ldots, \tau_{i_{n}} \gamma_{n}\right\rangle_{d}, \gamma_{i} \in R$, полностью определяются коэффициентами $I-р я д а I^{X}$.

Кроме того, есть еще один способ найти двухточечные инварианты, зная лишь свободный член $I$-ряда. Продемонстрируем его на примере трехмерного многообразия.

Рассмотрим гладкое трехмерное многообразие Фано $Y$ ранга 1 и индекса 1. Положим $I_{H^{0}}^{Y}=1+d_{2} q^{2}+d_{3} q^{3}+d_{4} q^{4}+d_{5} q^{5}+d_{6} q^{6}+\cdots$. Рассмотрим отображение $f: \mathbb{A}^{5} \rightarrow \mathbb{A}^{5}$, задаваемое многочленами $f_{i-2,3}^{i}$, соответствующими инвариантам $\left\langle\tau_{i-2} p t\right\rangle_{i}=d_{i}$ для $i=2, \ldots, 6$.

\section{ПрЕДЛОЖЕНИЕ 6.2.2. Отображение $f$ бирационалъно.}

ДокАзАтЕльство. Предложение проверяется непосредственным вычислением. Заметим, что это отображение бирегулярно, если

$$
-495 d_{3} d_{5}+261 d_{2} d_{3}^{2}-312 d_{4} d_{2}^{2}+432 d_{4}^{2}+56 d_{2}^{4} \neq 0,
$$

что (апостериори) верно для гладких трехмерных многообразий Фано ранга 1.

6.3. Гипотеза Голышева. Рассуждения, изложенные в этой статье, позволяют найти считающие матрицы для полных пересечений в проективных пространствах (восстановленные Голышевым в [32] по соответствующим уравнениям $D 3$ из теоремы Гивенталя в [4]) и матрицу для многообразия $V_{5}$, полученную Бовилем в [25], и доказать гипотезу Голышева для них. Также можно показать ее справедливость для $V_{12}$ (сечения ортогонального грассманиана $\mathrm{OG}(5,10)$ линейным подпространством коразмерности 7$), V_{16}$ (сечения лагранжева грассманиана $\operatorname{LG}(3,6)$ линейным подпространством коразмерности 3$)$ и $V_{18}$ (сечения грассманиана группы $G_{2}$ линейным подпространством коразмерности 2). Квантовое умножение на дивизор для грассманианов этих групп описано в [33]. Свидетельство того, что гипотеза для этих многообразий верна, получено В. Голышевым. Считающая матрица многообразия $V_{22}$ найдена А. Кузнецовым; она также согласуется с гипотезой. Наконец, считающие матрицы накрытия $\mathbb{P}^{3}$ с ветвлением в квартике, двойного накрытия $\mathbb{P}^{3}$ с ветвлением в секстике и двойного накрытия конуса над поверхностью Веронезе степени 4 с ветвлением в кубике можно также найти по этому методу. Для этого воспользуемся их описанием как гиперповерхностей во взвешенных проективных пространствах и найдем их $I$-ряд, обобщив формулу Гивенталя для гладких полных пересечений в торических многообразиях на случай полных пересечений в особых торических многообразиях.

\section{4. Случай числа Пикара, большего 1.}

ЗАмЕчАНИЕ 6.4.1. Все методы для многообразий с числом Пикара, равным 1, описанные выше, без труда обобщаются на случай многообразий с числом Пикара, большим 1. Вместо степени в этом случае необходимо ввести 
мультистепень $d=\left(d_{1}, \ldots, d_{r}\right)$, вместо переменной $q$ надо ввести многомерную переменную $q=\left(q_{1}, \ldots, q_{r}\right)$ и т.д.

Автор благодарит В. Голышева за тесное обсуждение этой работы, А. Гатманна за полезные советы, А. Гивенталя за ссылку на гипотезу Хори-Вафа, Д. Орлова, И. Чельцова и К. Шрамова за комментарии при подготовке работы.

\section{Список литературы}

[1] M. Kontsevich, Yu. Manin, "Gromov-Witten classes, quantum cohomology, and enumerative geometry", Comm. Math. Phys., 164:3 (1994), 525-562.

[2] K. Behrend, Yu. Manin, "Stacks of stable maps and Gromov-Witten invariants", Duke Math J., 85:1 (1996), 1-60; arXiv: alg-geom/9506023.

[3] K. Behrend, "Gromov-Witten invariants in algebraic geometry", Invent. Math., 127:3 (1997), 601-617.

[4] A. B. Givental, "Equivariant Gromov-Witten invariants", Int. Math. Res. Not., 1996:13 (1996), 613-663; arXiv: alg-geom/9603021.

[5] A. Gathmann, "Relative Gromov-Witten invariants and the mirror formula", Math. Ann., 325:2 (2003), 393-412; arXiv: math. AG/0009190.

[6] A. Bertram, I. Ciocan-Fontanine, B. Kim, "Two proofs of a conjecture of Hori and Vafa", Duke Math. J., 126:1 (2005), 101-136; arXiv: math.AG/0304403.

[7] A. Bertram, H. P. Kley, "New recursions for genus-zero Gromov-Witten invariants", Topology, 44:1 (2005), 1-24; arXiv: math.AG/0007082.

[8] Y.-P. Lee, R. Pandharipande, "A reconstruction theorem in quantum cohomology and quantum K-theory", Amer. J. Math., 126:6 (2004), 1367-1379; arXiv: math.AG/ 0104084.

[9] Н.П. Гушель, "О многообразиях Фано рода 6", Изв. АН СССР. Сер. матем., 46:6 (1982), 1159-1174; англ. пер.: N. Gushel', "On Fano varieties of genus 6", Math. USSR-Izv., 21:3 (1983), 445-459.

[10] Н. П. Гушель, “О многообразиях Фано рода 8”, УМH, 38:1 (1983), 163-164; англ. пер.: N. Gushel', "Fano varieties of genus 8", Russian Math. Surveys, 38:1 (1983), 192-193.

[11] Н. П. Гушель, "О трехмерных многообразиях Фано рода 8", Алгебра и анализ, 4:1 (1992), 120-134; англ. пер.: N. Gushel', "On Fano 3-folds of genus 8", St. Petersburg Math. J., 4:1 (1993), 115-129.

[12] В. А. Исковских, Лекции по трехмерным алгебраическим многообразиям. Многообразия Фано, Изд-во МГУ, М., 1988.

[13] В.А. Исковских, "Антиканонические модели трехмерных алгебраических многообразий”, Итоги науки и техники. Соврем. проблемы матем., 12, ВИНИТИ, M., 1979, 59-157; англ. пер.: V.A. Iskovskikh, "Anticanonical models of threedimensional algebraic varieties", J. Soviet Math., 13 (1980), 745-814.

[14] A. Iliev, "Geometry of the Fano threefold of degree 10 of the first type", Curves, Jacobians, and Abelian varieties (Amherst, AM, 1990), Contemp. Math., 136, Amer. Math. Soc., Providence, RI, 1992, 209-254.

[15] V. Iskovskikh, Yu. Prokhorov, Fano varieties, Algebraic geometry V, Encyclopaedia Math. Sci., 47, Springer, Berlin, 1999.

[16] D. Logachev, Fano threefolds of genus 6, arXiv: math.AG/0407147.

[17] D. Yu. Logachev, "Birational isomorphism of a three-dimensional cubic and of the section of $G(2,6)$ by five hyperplanes, and similar problems", The birational geometry of algebraic varieties, Sbornik Nauchnykh Trudov, 203, Yaroslav. Gos. Ped. Inst., Yaroslavl', 1983, 39-45. 
[18] Sh. Mukai, "Fano 3-folds", Complex projective geometry, London Math. Soc. Lecture Note Ser., 179, Cambridge Univ. Press, Cambridge, 1992, 255-263.

[19] Д. Г. Маркушевич, "Численные инварианты семейств прямых на некоторых многообразиях Фано", Матем. сб., 116(158):2(10) (1981), 265-288; англ. пер.: D. G. Markuševič, "Numerical invariants of families of lines on some Fano varieties", Math. USSR-Sb., 44:2 (1983), 239-260.

[20] A. Bayer, Yu. I. Manin, "(Semi)simple exercises in quantum cohomology", The Fano conference, Univ. Torino, Turin, 2004, 143-173; arXiv: math.AG/0103164.

[21] G. Ciolli, "On the quantum cohomology of some Fano threefolds and a conjecture of Dubrovin", Internat. J. Math., 16:8 (2005), 823-839; arXiv: math. AG/0403300.

[22] V. Ancona, M. Maggesi, "On the quantum cohomology of Fano bundles over projective spaces", The Fano conference, Univ. Torino, Turin, 2004, 81-98; arXiv: math.AG/ 0012046.

[23] V.V. Batyrev, "Quantum cohomology rings of toric manifolds", Journées de Géométrie Algébrique d'Orsay (Orsay, 1992), Astérisque, 218, 1993, 9-34; arXiv: alg-geom/9310004.

[24] Z. Qin, Y. Ruan, "Quantum cohomology of projective bundles over $\mathbb{P}^{n}$ ", Trans. Amer. Math. Soc., 350:9 (1998), 3615-3638.

[25] A. Beauville, "Quantum cohomology of complete intersections", Mat. Fiz. Anal. Geom., 2:3-4 (1995), 384-398; arXiv: alg-geom/9501008.

[26] В. В. Голышев, "Проблемы геометричности и модулярность некоторых вариаций Римана-Роха", Докл. РАН, 386:5 (2002), 583-588; англ. пер.: V. V. Golyshev, "The geometricity problem and modularity of certain Riemann-Roch variations", Dokl. Math., 66:2 (2002), 231-236.

[27] Ю.И. Манин, Фробениусовы многообразия, квантовые когомологии и пространства модулей, Факториал, М., 2002; Yu. I. Manin, Frobenius manifolds, quantum cohomology, and moduli spaces, Amer. Math. Soc. Colloq. Publ., 47, Amer. Math. Soc., Providence, 1999.

[28] A. Vistoli, "Intersection theory on algebraic stacks and on their moduli spaces", Invent. Math., 97:3 (1989), 613-670.

[29] H. Hori, C. Vafa, Mirror symmetry, arXiv: hep-th/0002222.

[30] A. Gathmann, "Absolute and relative Gromov-Witten invariants of very ample hypersurfaces", Duke Math. J., 115:2 (2002), 171-203; arXiv: math. AG/9908054.

[31] M. Kontsevich, Yu. Manin, "Relations between the correlators of the topological sigma-model coupled to gravity", Comm. Math. Phys., 196 (1998), 385-398.

[32] В. В. Голышев, "Модулярность уравнений D3 и классификация В. Исковских", Докл. РАН, 396:6 (2004), 733-739; англ. пер.: V. V. Golyshev, "Modularity of equations D3 and the Iskovskikh classification", Dokl. Math., 69:3 (2004), 443-449; arXiv: math. AG/0405039.

[33] W. Fulton, C. Woodward, "On the quantum product of Schubert classes", J. Algebraic Geom., 13:4 (2004), 641-661; arXiv: math.AG/0112183.

В. В. Пржиялковский (V. V. Przyjalkowski)

Математический институт им. В. А. Стеклова РАН

E-mail: victorprz@mi.ras.ru
Поступила в редакцию 13.07.2004 и 20.04.2006 